\title{
Inclusión de Estudiantes Migrantes en la Región de La Araucanía, Chile: Representaciones desde los Directivos Escolares
}

\section{Inclusion of Migrant Students in the Region of La Araucanía, Chile: Representations from School Leaders}

\author{
Omar Aravena Kenigs *1 \\ Paula Riquelme Bravo ${ }^{2}$ \\ $\mathrm{M}^{\mathrm{a}}$ Elena Mellado Hernández ${ }^{1}$ \\ Carolina Villagra Bravo ${ }^{1}$ \\ ${ }^{1}$ Centro de Desarrollo de Liderazgo Educativo, Universidad Católica de Temuco, Chile \\ ${ }^{2}$ Universidad Andrés Bello, Chile
}

\begin{abstract}
El creciente fenómeno migratorio que vive Chile, sumado a la diversidad étnica y cultural propia del territorio, desafía a los líderes escolares a repensar escenarios educativos que favorezcan los procesos de inclusión y justicia socia del estudiantado. En este contexto, el objetivo del presente artículo es describir las representaciones de docentes directivos acerca de la inclusión de estudiantes migrantes en centros educativos de la región de La Araucanía, Chile. La investigación utiliza un método mix to y posee un alcance descriptivo. Los participantes son 79 directores y directoras de escuelas y liceos subvencionados por el Estado. Los resultados evidencian que los líderes escolares asumen la inclusión escolar desde un enfoque técnico y desarrollan prácticas focalizadas principalmente en dar cumplimiento a la normativa de la Ley de Inclusión, por sobre la implementación de prácticas orientadas a favorecer el desarrollo profesional docente y el aprendizaje del estudiantado. Las conclusiones destacan la necesidad de transitar hacia liderazgos más inclusivos y críticos, capaces influenciar prácticas sustentadas en el reconocimiento y valoración de las diferencias del estudiantado como oportunidad para favorecer el proceso de enseñanzaaprendizajes desde un enfoque inclusivo e intercultural.
\end{abstract}

Descriptores: Liderazgo; Inclusión; Interculturalidad; Estudiantado; Migración.

Chile's increasing migration phenomenon, added to the ethnic and cultural diversity of the territory, challenges school leaders to rethink educational stages that fortify the processes of inclusion and social justice. In this context, the aim of this article is to describe the representations of senior teachers about the inclusion of migrant students in schools in La Araucanía region, Chile. The research uses an assorted method and has a descriptive coverage. The participants are 79 directors of schools and high-schools subsidized by the State. The results evidence that school leaders assume school inclusion from a technical approach and develop practices mainly focused on accomplish with the regulations of the Inclusion Law, above the implementation of practices aimed to assist the professional development of teachers and student's learning. The conclusions highlight the need to move towards more inclusive and critical leaderships, capable of influencing practices based on the recognition and assessment of student's differences as an opportunity to assist the teaching-learning process and promote professional teacher development.

Keywords: Leadership; Inclusion; Interculturality; Student; Migration.

*Contacto: oaravena@uct.cl

ISSN: 0718-7378

www.rinace.net/rlei/
Recibido: 03/12/2018

$1^{\text {a }}$ Evaluación: 20/01/2019

Aceptado: 12/03/2019 


\title{
Introducción
}

Desde el año 2016 entra en vigencia en Chile la Ley de Inclusión Escolar ( $n^{\circ}$ 20.845), cuyo principal propósito es terminar gradualmente con el lucro en todos los establecimientos que reciben subvención del Estado y poner fin a la selección arbitraria de estudiantes en el sistema educativo (MINEDUC, 2017a). Esta Ley representa uno de los pilares de la reforma educativa y su implementación conlleva la necesidad de superar la tradicional concepción de inclusión escolar, asociada a la integración de niños, niñas y jóvenes con necesidades educativas especiales, para avanzar hacia un enfoque más amplio y comprensivo, sustentado en el reconocimiento y valoración de las diferencias durante el proceso de enseñanza y aprendizaje (Dovigo, 2014; Leiva, 2013; Mellado et al., 2017; Sánchez y Gil, 2015). En relación a lo anterior, la Ley de Inclusión Escolar es enfática en señalar:

\begin{abstract}
El sistema propenderá a eliminar todas las formas de discriminación que impidan el aprendizaje y la participación de los y las estudiantes. Asimismo, propiciará que los establecimientos educativos sean un lugar de encuentro entre estudiantes de distintas condiciones socioeconómicas, culturales, étnicas, de género, nacionalidad o religión. (Art. $1^{\circ}$, numeral 1 , letra e)
\end{abstract}

La puesta en marcha de la Ley de Inclusión Escolar sumado al significativo aumento de la diversidad social y cultural del país, vislumbra grandes desafíos y oportunidades para los equipos directivos encargados de liderar las comunidades educativas. (MINEDUC, 2017b). Entre los desafíos, destaca la necesidad de reflexionar acerca de las barreras culturales y estructurales que generan y validan prácticas de exclusión y segregación al interior de los centros educativos (Gairín, 2014; Lehtomäki, Moate y Posti-Ahokas, 2015). Por otra parte, el actual escenario hace más latente que nunca la oportunidad de desplegar liderazgos desde un enfoque inclusivo que contribuyan a eliminar las brechas que obstaculizan el buen aprendizaje de los y las estudiantes (Murillo y Duk, 2017; RincónGallardo y Fullan, 2016; Ryan, 2016).

Muntaner (2010) plantea que transitar hacia una educación inclusiva con enfoque intercultural requiere un cambio radical, no solo a nivel de políticas públicas, sino también en las representaciones e imaginarios de los actores educativos encargados de implementarlas. En este sentido, el liderazgo escolar emerge como un factor que puede condicionar fuertemente la construcción de culturas escolares inclusivas (Alavez 2014; Booth, 2010; Echeita, 2013; MINEDUC, 2016a; Ossa et al., 2014) y es considerado como la segunda variable intraescuela que incide en los resultados de aprendizaje del estudiantado (Anderson, 2010; Barber y Mourshed, 2007; Leithwood et al., 2006).

El presente artículo es parte de los resultados de un proyecto de investigación financiado por el MINEDUC y ejecutado por un equipo del Centro de Desarrollo de Liderazgo Educativo (CEDLE). El principal objetivo del estudio fue analizar las representaciones de los directivos escolares acerca de la inclusión de estudiantes migrantes en escuelas y liceos de la región de La Araucanía, Chile. Es importante destacar que esta región posee amplia diversidad étnica y cultural, característica que se acentúa con la creciente presencia de estudiantes migrantes en las aulas. Por tanto, profundizar en las representaciones de los líderes escolares permitirá identificar los principales desafíos en materia de liderazgo para avanzar hacia la construcción de culturas escolares inclusivas que aseguren oportunidades de aprendizaje de calidad para todos y todas sus estudiantes. 


\section{Estudiantes migrantes en Chile: Hacia la construcción de escuelas inclusivas con enfoque intercultural}

Según datos estadísticos proporcionados por el MINEDUC (2018) la matrícula de niños, niñas y jóvenes extranjeros en escuelas y liceos del país alcanzó los 113.585 estudiantes durante el año 2018. Esta cifra equivale al 3,2\% de matrícula total de los establecimientos municipales, concentrándose el 59,3\% en la Región Metropolitana. En lo que refiere a la región de La Araucanía, el censo 2017 constató un total de 957.224 personas, de las cuales 15.616 son extranjeras, representando el 1,6\% del total regional. En el contexto educativo, el informe del Instituto Nacional de Derechos Humanos (2018) da cuenta que los estudiantes extranjeros que asisten a escuelas municipales, solo en la capital regional de la región de La Araucanía llegó a 250 estudiantes durante el año 2018.

Frente al explosivo aumento de las cifras de estudiantes extranjeros, el año 2015 la entonces presidenta de Chile, Michelle Bachelet, presentó un documento oficial con lineamientos para el diseño de una Política Nacional Migratoria, basada en un enfoque transversal de derechos humanos y de género, sustentado en el principio de no discriminación. De esta forma, la política educativa ha demostrado importantes avances en generar condiciones estructurales para asegurar el derecho a la educación de todo el estudiantado migrante. De esta forma, las comunidades escolares enfrentan el desafío de propiciar culturas inclusivas que velen por el respeto y cumplimiento de este derecho (Cerón, Pérez-Alvarado y Poblete, 2017; Fernández, 2018; Jiménez et al., 2017; Mellado y Chaucono, 2016).

En los últimos años, la investigación sobre inclusión educativa ha puesto su foco en la interculturalidad (Bustos y Gairín, 2017; Diez, 2013; Joiko y Vásquez, 2016; Riedemann y Stefoni, 2016; Sales, Fernández y Moliner, 2012). Este enfoque busca la construcción de diálogos horizontales entre distintas culturas que comparten un territorio y espacio social, donde el reconocimiento de las diferencias individuales es el primer paso para comprender la diversidad desde un plano cognitivo, emocional y valórico (Leiva, 2013). En atención a este punto, Tubino (2011) profundiza en dos perspectivas de interculturalidad. La primera, denominada interculturalidad funcional, promueve el diálogo y la tolerancia entre los distintos grupos socioculturales, poniendo el foco en cuestiones identitarias, pero sin alterar las relaciones de poder existentes. Por otra parte, el autor plantea la existencia de una perspectiva intercultural crítica, que cuestiona las diferencias y desigualdades entre diferentes grupos socioculturales, con la finalidad de construir relaciones más igualitarias. En consecuencia, avanzar hacia un enfoque de educación intercultural significa asumir también, desde el liderazgo, una actitud de apertura en la forma de relacionarse en contextos de diversidad cultural.

En el contexto educativo, autores como Jiménez (2014) y Apablaza (2015) plantean la necesidad de superar aquellos paradigmas tradicionales de liderazgo que aún prevalecen en las escuelas y que validan prácticas donde predomina la asimilación y adaptación del estudiantado a la cultura escolar dominante. En Chile, la Superintendencia de Educación (MINEDUC, 2016b) identificó entre las principales barreras para el aprendizaje la ausencia de herramientas metodológicas y flexibilidad curricular para reconocer y valorar la diversidad presente en las aulas. Por su parte, Quintriqueo y otros (2016) advierten que este carácter mono-cultural de la escuela y condiciona las prácticas de enseñanza, dificultando una adecuada gestión de la diversidad social y cultural del estudiantado. 
Por consiguiente, el cambiante y cada vez más complejo escenario educativo requiere el desarrollo de ciertas competencias específicas en los líderes escolares; y exige también cambios en la forma de gestionar las relaciones al interior de la escuela (Jiménez y Fardella, 2015; Mampaey y Zanoni, 2015; Martínez et al., 2015; Rojas y Armijo, 2016). En otras palabras, se precisan líderes capaces de generar condiciones que posibiliten el aprendizaje que todos estudiantes contexto socialmente justo y democrático (Hargreaves y Fullan, 2004; González-González, 2008). El siguiente apartado presenta una revisión de las características de los líderes inclusivos y sus prácticas para favorecer la transformación cultural y el desarrollo de los integrantes de la comunidad.

\section{Liderazgo directivo para la inclusión educativa y justicia social}

Diferentes estudios han logrado caracterizar el perfil de aquellos líderes que favorecen la inclusión y la justicia social en los centros educativos. Echeita y Ainscow (2011) reconocen al líder inclusivo como aquel que privilegia las relaciones humanas y visualiza a la comunidad educativa como un sistema abierto de relaciones. Otros autores enfatizan en que un líder para la justica social es altamente dialógico y democrático, desarrolla su gestión en un marco de colaboración, respeto y valoración de las diferencias (Calvo, Verdugo y Amor, 2016; Essomba, 2006; Hernández-Castilla, Euán e Hidalgo, 2013). Por su parte, Murillo y Hernández-Castilla (2011) aportan diciendo que un líder para justicia social focaliza en la construcción de una organización educativa que trabaja en contra de las desigualdades y por el desarrollo de una sociedad más justa.

Es importante mencionar que el liderazgo para la justicia social no es responsabilidad exclusiva de las personas que poseen un cargo formal en las organizaciones escolares, sino que es un ejercicio colectivo entre individuos que comparten una visión o proyecto (Booth y Ainscow, 2015; Murillo et al., 2010; Tintore-Espuny, 2018). Por tanto, los líderes inclusivos no adoptan un estilo único de liderazgo, sino que despliegan una mixtura de enfoques que buscan el bien común, respeto por la cultura de los estudiantes, sus familias y la dignidad de las personas. Entre los enfoques de liderazgo para la justicia social, la literatura destaca el liderazgo sostenible (Hargreaves y Fink, 2008); liderazgo sistémico (Sanders, 2012); liderazgo distribuido (Spinalle y Diamond, 2007); liderazgo pedagógico (Bolívar y Murillo, 2017; Robinson, 2016); liderazgo transformacional (Sun y Leithwood, 2015); liderazgo ético (Giles y Cuellar, 2016), entre otros.

Algunos autores, reconocen algunas prácticas propias del liderazgo para la inclusión y justicia social. Leithwood y Riehl (2005) dicen que los líderes inclusivos articulan una visión compartida de escuela con los integrantes de la comunidad; promueven la colaboración entre escuela, familia y comunidad local y desarrollan continuamente a las personas para fortalecer la cultura inclusiva al interior de las escuelas. Por su parte, autores como Arar (2015) y Bolívar (2012) destacan la capacidad de estos líderes para ayudar a los estudiantes a desarrollar todo su potencial y motivan constantemente al profesorado para lograr esta meta (Mintrop y Órdenes, 2017).

Cabe destacar que la política educativa de liderazgo escolar en Chile, también ha enfatizado en orientar el desarrollo de prácticas inclusivas al interior de los centros escolares. El MINEDUC (2015) diseñó un nuevo Marco para la Buena Dirección y el Liderazgo Escolar, el cual destaca en varias de sus dimensiones una serie de habilidades, 
principios y conocimientos profesionales que los líderes escolares deben poseer para desarrollar prácticas directivas que aseguren el aprendizaje integral de todos los estudiantes en un ambiente de sana convivencia (cuadro 1).

Cuadro 1. Síntesis de prácticas inclusivas propuestas

\section{DIMENSIÓN PRÁCTICAS DE LIDERAZGO PARA LA INCLUSIÓN}

Definen o revisan, en conjunto con su comunidad educativa, el proyecto educativo institucional y curricular, enfocado en el mejoramiento de los logros de aprendizajes de todos los

Construyen e

Implementan una visión estratégica compartida: estudiantes, así como en los valores de la equidad, la inclusión y el respeto a la diversidad.

Promueven y modelan activamente una cultura escolar inclusiva, equitativa y de altas expectativas sobre los logros de aprendizaje de los estudiantes y desempeño de todos los miembros del establecimiento.

Aseguran la implementación de estrategias para identificar y apoyar tempranamente a los estudiantes que presenten dificultades en los aprendizajes o en los ámbitos conductual, afectivo o social.

Lideran los procesos de enseñanza y aprendizaje

Identifican y difunden, entre sus docentes y directivos, buenas prácticas de enseñanza y aprendizaje como de gestión pedagógica, tanto internas como externas y que respondan a las necesidades de sus estudiantes.

Desarrollan e implementan una política que asegura una cultura inclusiva y las condiciones para que las personas se traten de manera equitativa, justa, con dignidad y respeto, resguardando los derechos y deberes de la comunidad escolar.

Gestionan la convivencia y la participación de la

Desarrollan y mantienen relaciones de comunicación y colaboración permanente con los padres y apoderados del establecimiento, con el objetivo de involucrarlos en los procesos formativos de los estudiantes

Generan oportunidades de participación y colaboración de los actores de la comunidad escolar a través de espacios formales, a fin de consolidar el logro de un clima escolar positivo y los objetivos expresados en el Proyecto Educativo Institucional.

Aseguran que el funcionamiento del establecimiento responda a las normas legales y las políticas educativas nacionales y locales.

Desarrollan y gestionan

En conjunto con el sostenedor, aseguran la disponibilidad de los el establecimiento escolar recursos requeridos por el establecimiento y los gestionan eficientemente, de manera de maximizar su uso en los procesos pedagógicos y el logro de las metas institucionales

Fuente: Elaborado a partir de MINEDUC (2015).

Es importante preciar que las orientaciones de la política educativa por sí solas no pueden generar transformación (Martínez et al., 2015). Aquí emerge la relevancia de asumir un fuerte liderazgo ético (Giles y Cuéllar, 2017) basado en una genuina preocupación por los demás y de orientar el propósito moral de la escuela hacia el desarrollo de los estudiantes y de la comunidad, por sobre la lógica de eficacia basado en la rendición de cuentas. Los líderes éticos mantienen un constante diálogo acerca de los propósitos de la educación y se esfuerzan por influenciar a los principios, creencias y valores de los integrantes de la comunidad hacia la búsqueda del bien común (Starratt, 2004).

En síntesis, avanzar hacia la construcción de culturas escolares inclusivas, requiere un esfuerzo conjunto entre las políticas educativas y las actitudes de los actores del sistema educativo (Murillo y Duk, 2016). En este sentido, es de relevante importancia desarrollar liderazgos que favorezcan el desarrollo de prácticas orientadas al reconocimiento y 
valoración de la diversidad, como un principio inherente al aprendizaje. Esto implica "abrir la escuela" hacia diálogos y profunda reflexión y colaboración entre los diversos actores educativos, en un marco de valores compartidos y fuerte compromiso moral por el aprendizaje de todos y todas las estudiantes como eje central de la escuela.

\section{Método}

El presente estudio se enmarca en una investigación educativa de alcance descriptivo. Utiliza una metodología mixta, con predominancia del enfoque cualitativo (cual $\rightarrow$ cuan).

\section{Participantes}

Los participantes del estudio son 79 directivos escolares, pertenecientes a 43 centros municipales (33 escuelas y 10 liceos) y 36 centros particulares subvencionados ( 28 escuelas y 8 liceos) de la región de La Araucanía, Chile. Los directivos son 31 hombres y 48 mujeres, cuya edad promedio alcanza los 51,3 años. La selección de los participantes fue de tipo no probabilística, considerando solo aquellos centros escolares que cuenten con al menos cinco estudiantes migrantes.

\section{Instrumentos de recolección de información}

Los instrumentos de recolección de información utilizados fueron un cuestionario cuantitativo, tipo escala Likert, aplicado en modalidad online a 79 directivos escolares. Este cuestionario indaga en las representaciones de los directivos escolares acerca de la inclusión educativa del estudiantado migrante. La estructura del instrumento se compone de 12 reactivos, agrupados en 3 dimensiones de análisis: 1) representaciones de directivos acerca de las prácticas de gestión y liderazgo; 2) representaciones de directivos acerca del rol del profesorado; 3) representaciones de directivos acerca del estudiantado migrantes.

Para cada uno de los ítems presentados, los participantes debieron puntuar cada aseveración, según mejor represente su parecer, en consideración a la siguiente escala: 1 (Total desacuerdo), 2 (Desacuerdo), 3 (Acuerdo), y 4 (Total acuerdo).

Posteriormente, se profundizó en forma cualitativa por medio de una entrevista semiestructurada, en las representaciones de 20 directivos escolares respecto a los distintos enfoques de inclusión educativa. En estas entrevistas se resguardó representatividad de género de los participantes (10 hombres y 10 mujeres) y representatividad de centros escolares municipales y particulares subvencionados.

\section{Validez de los instrumentos}

Para el diseño de los ítems y delimitación de las dimensiones del instrumento cuantitativo, se consideró la revisión y análisis de investigaciones referentes a inclusión educativa, migración, e interculturalidad en contextos escolares. La validez de contenido se realizó por juicio de expertos, quienes valoraron relevancia, pertinencia y claridad de cada ítem. Para validez de constructo se utilizó análisis factorial confirmatorio, mediante la técnica de extracción de factores con análisis de componentes principales y rotación VARIMAX. El resultado fue la confirmación de tres dimensiones o componentes que explican el 74,2\% acumulado de la varianza. Finalmente, el análisis de fiabilidad se calculó con el coeficiente Alfa de Cronbach, resultado un valor de .901 para el total de la escala (cuadro 2), resultado que otorga garantías científicas para su aplicación. 
Cuadro 2. Análisis de fiabilidad Alfa de Cronbach para las dimensiones del instrumento

\begin{tabular}{lcc}
\hline \multicolumn{1}{c}{ FACTORES } & $\mathbf{N}^{\mathbf{0}}$ REACTIVOS/ÍTEMS & $\begin{array}{c}\text { CoNSISTENCIA } \\
\text { INTERNA }\end{array}$ \\
\hline 1. Prácticas de gestión y liderazgo & 4 & 0,842 \\
2. Rol del profesorado & 4 & 0,912 \\
3. Desempeño del estudiantado migrante & 4 & 0,832 \\
\hline Total & 12 & 0,901 \\
\hline
\end{tabular}

Fuente: Elaboración propia.

\section{Análisis de la información}

El análisis de la información cuantitativa se realizó utilizando estadística descriptiva, basada en medidas de tendencia central y distribución porcentual. La data verbal fue analizada a través de la técnica de análisis de contenido, considerando categorías prestablecidas y emergentes.

\section{Resultados}

A continuación, se presentan los resultados del estudio en función de cada una de las dimensiones de la encuesta aplicada en la fase cuantitativa. Para una mayor comprensión de estos hallazgos, se complementa con el análisis de las categorías que emergen de las entrevistas en profundidad realizadas en la fase cualitativa.

3.1. Resultados Dimensión 1 del cuestionario: "Representaciones de los directivos escolares acerca de las prácticas de liderazgo para favorecer la inclusión del estudiantado migrante"

1. Reformulación de sellos y proyecto educativo institucional

2. Perfeccionamiento constante al profesorado y asistentes de la educación

3. Jornadas de reflexión con integrantes de la comunidad escolar

4. Actualización de reglamentos y protocolos internos

\section{$\square$ Total desacuerdo $\square$ Desacuerdo $\square$ Acuerdo $\square$ Total acuerdo}

Figura 1. Resultados de la dimensión "Representaciones de las prácticas de liderazgo" Fuente: Elaboración propia.

La figura 1 muestra que el $55 \%$ de los directivos encuestados considera que la reformulación de los proyectos educativos (ítem 1) y la actualización de reglamentos internos (ítem 4) son prácticas que han favorecido el proceso de inclusión del estudiantado migrante en sus centros escolares. Por otra parte, el $46 \%$ de los directivos escolares valora positivamente el desarrollo de jornadas reflexivas acerca de la inclusión escolar con los integrantes de la comunidad educativa (ítem 3). Por último, solo el 35\% de los directivos escolares considera el perfeccionamiento y formación docente como una práctica relevante para favorecer la inclusión del estudiantado. 
En esta dimensión, demuestra que los directivos escolares valoran altamente las prácticas asociadas a la actualización y reformulación de los instrumentos de gestión, como una estrategia que contribuir a la inclusión educativa del estudiantado. Sin embargo, cuando analizamos los relatos aportados por las entrevistas, se evidencia una racionalidad técnica de tipo instrumental (Mansilla y Beltrán, 2016), relativas al asegurar comportamientos desde un orden técnico, reglamentario y normativo. A continuación, se exponen ejemplo de este enfoque.

Categoría "Liderazgo para la inclusión desde una racionalidad instrumental":

Con la incorporación de estudiantes extranjeros hemos tenido que actualizar reglamentos y manuales de convivencia. Por ejemplo, estipular sanciones y procedimientos para evitar que estos niños sean molestados o discriminados. (Directora, caso 4)

Nuestros supervisores técnicos del MINEDUC, nos sugirieron que debemos actualizar el plan de inclusión y los reglamentos, porque, además la agencia o la superintendencia, los pueden solicitar al momento de las visitas al colegio. (Director, caso 9)

Desde esta perspectiva técnica, los líderes escolares tratan normar las relaciones desde dispositivos de control, omitiendo los procesos formativos y reflexivos en torno a la inclusión escolar de estudiantes migrantes. Este enfoque, según Tubino (2005) dificultaría avanzar hacia un enfoque crítico de la interculturalidad. Sin embargo, en el discurso de los directivos escolares, también emergen perspectivas más críticas, centradas en favorecer las relaciones y el reconocimiento y valoración de la diversidad, los que se describen a continuación:

Categoría "Liderazgo para la inclusión desde una racionalidad crítica":

Los protocolos de convivencia han sido modificados a favor de las familias extranjeras...con el equipo directivo y los profesores nos dimos cuenta varios aspectos que no estábamos considerando. Por ejemplo, cuando llegan los estudiantes y sus familias ¿̇quién las asesora, las acoge y se preocupa de ver cuáles son sus necesidades? Todo eso ahora está considerado y hay equipos destinados a este apoyo. (Director, caso 2)

Nosotros tuvimos que modificar nuestro proyecto educativo, con la llegada de los estudiantes migrantes, tuvimos replantear nuestra visión y misión que estaba pensada en la interculturalidad desde la perspectiva de la cultura mapuche...pero ahora, imaginese, tenemos estudiantes de nueve países diferentes y nuestro proyecto tiene que dar respuesta a todos. (Directora, caso 12)

En la anterior categoría de análisis, se explicitan relatos de líderes escolares que cuestionan sus prácticas desde una postura crítica, que supera la mirada asociada a solo cumplir con la normativa legal. En estos relatos, se devela que los directivos escolares centran su foco en generar procesos que aseguren bienestar de los estudiantes y sus familias (Rodríguez-Ruiz, Martínez-González y López, 2016), así también, cuestionan el proyecto educativo en función del reconocimiento de las culturas presentes en la escuela.

\subsection{Resultados dimensión 2: "Representaciones directivas acerca de las prácticas docentes para dar respuesta a la inclusión del estudiantado migrante”}

La figura 2 muestra que el $60 \%$ de los directivos encuestados considera que el profesorado de su centro escolar posee una actitud favorable hacia la inclusión educativa (ítem 5). Un porcentaje similar de directivos piensa que el profesorado reflexiona constantemente acerca de sus prácticas para atender la diversidad en aula (ítem 7). En cuento a las prácticas 
docentes, el $66 \%$ de los directivos considera que el profesorado diversifica la enseñanza para favorecer el aprendizaje (ítem 6) y el 57\% de los directivos posee la convicción que los docentes consideran los saberes culturales del estudiantado migrante durante el proceso de enseñanza y aprendizaje (ítem 8).

5. El profesorado posee una actitud favorable hacia la inclusión

$6 \mathrm{El}$ profesorado diversifica sus actividades de enseñanza

7. El profesorado reflexiona constantemente sobre sus prácticas de enseñanza

8. El profesorado incorpora los saberes culturales del estudiantado migrante

\section{$\square$ Total desacuerdo}

$\square$ Desacuerdo

\begin{tabular}{|l|l|l|l|}
\hline $16 \%$ & $22 \%$ & $39 \%$ & $21 \%$ \\
\hline \multicolumn{5}{|c|}{$31 \%$} & $35 \%$ \\
\hline $15 \%$ & $17 \%$ & $35 \%$ & $25 \%$ \\
\hline \multicolumn{5}{|c|}{$35 \%$} & $17 \%$ \\
\hline $17 \%$ & $21 \%$ & $30 \%$ & $1 \%$ \\
\hline $19 \%$ & $22 \%$ & $40 \%$ & \\
\hline
\end{tabular}

$\mathrm{N}=79$

Figura 2. Resultados de la dimensión "Representaciones de las prácticas docentes" Fuente: Elaboración propia.

En esta dimensión, se aprecia que, si bien un alto porcentaje de directivos posee representaciones favorables respecto al desempeño de los docentes, existe casi un tercio de directores y directoras (alrededor del 30\%) que perciben las prácticas docentes como un factor obstaculizador para la inclusión en el aula. Estas cifras se tensionan también con los resultados obtenidos en la dimensión 1 del cuestionario, donde el $75 \%$ de los directivos escolares mostró baja valoración hacia el perfeccionamiento docente (ítem 2) como estrategia que puede impactar positivamente en los procesos de inclusión escolar.

$\mathrm{Al}$ profundizar en los relatos cualitativos de esta dimensión, se develan distintas formas de afrontar el desarrollo profesional docente del profesorado. En primer lugar, se exponen textualidades que evidencian directivos escolares con bajas expectativas acerca de las capacidades del profesorado para favorecer la inclusión del estudiantado migrante:

Categoría "Necesidades formativas del profesorado como un obstaculizador para la inclusión”

Con la ley de inclusión escolar, la verdad es que ahora todos hablan de inclusión, pero en realidad creo que la mayoría de los profesores no están preparados para trabajar con la diversidad, al menos yo no sé cómo entregarles herramientas para afrontar estos desafios, porque nadie los preparó para este escenario. (Director, caso 6)

En realidad, los profesores de mi escuela son muy tradicionales y cuesta que cambien algunas prácticas, por ejemplo, hay docentes que se reúsan a que los profesionales del PIE realicen co-docencia, y los siguen viendo como apoyo de un grupo de estudiantes en el aula. (Directora, caso 3)

En estos relatos, dan cuenta de la necesidad de asumir un fuerte liderazgo pedagógico por parte de los directivos escolares (Bolívar, 2012). En cuanto, actualmente son llamados a liderar el desarrollo de capacidades docentes y focalizar su quehacer en atender las necesidades formativas del profesorado para propiciar la mejora de los aprendizajes (MINEDUC, 2015). En atención a este desafío, también emergen relatos de prácticas directivas visualizan en inclusión educativa de los niños, niñas y jóvenes, una oportunidad para gestionar el aprendizaje profesional del profesorado: 
Categoría "Inclusión escolar como oportunidad para el desarrollo profesional docente"

Tenemos talleres de formación interna con los docentes porque sentimos que necesitamos prepararnos constantemente estamos para atender a estos niños. Junto al equipo directivo hemos buscado estrategias para hacer un trabajo efectivo, porque consideramos que la escuela debe avanzar paulatinamente en estos desafios. (Director, caso 10)

En la escuela hemos abierto los espacios para que los profesores, en primer lugar, se sensibilicen respecto a quienes son sus estudiantes...es evidente que los chiquillos de hoy han cambiado y no podemos hacer las mismas clases de hace 20 años atrás. Hay profesores que tiene mucha experiencia, algunos han hecho pasantías fuera de Chile y ellos, por ejemplo, han liderado estos espacios de reflexión, nos han ayudado a dar otra mirada a la escuela y a la forma de enseñar. (Directora, caso 1)

Como muestran estos relatos, gestionar el desarrollo profesional docente es un buen ejemplo de líderes inclusivos. Tal como planeta Calvo (2013), uno de los elementos que más incide en el proceso de aprendizaje de los estudiantes tiene que ver con lo que creen, pueden y están dispuestos a hacer los docentes, por tanto, el rol del liderar escolar es clave a la hora de influir positivamente en la motivación del profesorado (Mintrop y Órdenes, 2017).

\subsection{Resultados dimensión 3: "Representaciones directivas acerca de la inclusión educativa del estudiantado migrante"}

Los resultados de la dimensión 3 muestran que el $71 \%$ de los directivos escolares valora positivamente la acogida que los niñas y niños entregan a sus compañeros de origen extranjero. En este mismo sentido, el $61 \%$ de los directivos considera que la llegada de estudiantes migrantes ha favorecido la convivencia escolar en el establecimiento. Sin embargo, este porcentaje disminuye cuando se alude a los resultados educativos, puesto que el $43 \%$ directivos valora desfavorablemente el desempeño de los estudiantes migrantes en cuanto a sus resultados educativos (ítem 12). Esto se reafirma en el ítem 11, que arroja un $62 \%$ de directivos que piensa los estudiantes migrantes poseen dificultad para enfrentar el currículum escolar.

9. Los estudiantes migrantes son bien acogidos por sus compañeros

10. Los estudiantes migrantes favorecen la convivencia escolar

11. Los estudiantes migrantes poseen dificultad para responder al currículum nacional

12. Los estudiantes migrantes aportan a mejorar los resultados educativos

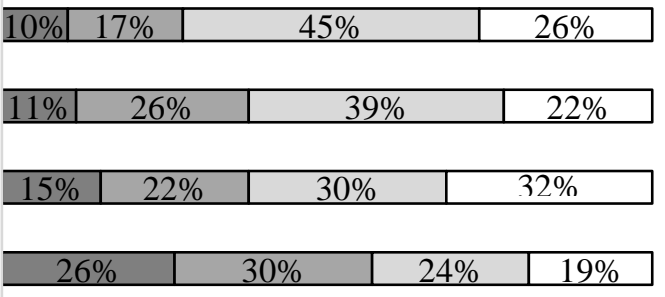

$\mathrm{N}=79$

\section{$\square$ Total desacuerdo $\square$ Desacuerdo $\square$ Acuerdo $\square$ Total acuerdo}

Figura 3. Resultados de la dimensión 1 "Representaciones de las prácticas de liderazgo"

Fuente: Elaboración propia.

Los resultados de esta dimensión, dan cuenta que un alto porcentaje de los directivos escolares posee representaciones del aprendizaje asociado al cumplimiento del currículum nacional por parte de los estudiantes. Este enfoque centra su atención en el resultado de objetivos de aprendizaje prescritos por sobre los saberes sociales y culturales del estudiantado. Esto se evidencia con mayor fuerza en los relatos de los directivos escolares: 
Categoría "Invisibilización de los saberes culturales del estudiantado"

Los estudiantes migrantes en realidad siempre vienen con sus aprendizajes bien disminuidos, les cuesta harto adaptarse a la escuela. Igual es difícil, porque llegan muchas veces a mitad de año, viene atrasados con los contenidos, otros poseen dificultad con el lenguaje, así que es un escenario bien complicado para ellos. (Directora, caso 14)

Es súper complicado nivelarlos, porque traen rezagos en los aprendizajes. Más aun, muchos de los contenidos son desconocidos para ellos. Lo otro que dificulta, es que los equipos multidisciplinarios siempre están copados, hay poco tiempo para una educación tan personalizada. (Director, caso 7)

Las expresiones descritas, son coherentes con la implementación del currículum desde una lógica monocultural, con foco en el logro de "contenidos" curriculares (Quintriqueo et al., 2016). Los relatos anteriores también dan cuenta de representaciones acerca de los estudiantes migrantes donde se "invisibilizan" sus saberes culturales y se valora solo los conocimientos que responden a la lógica del cumplimiento del currículum.

Sin embargo, también se aprecian líderes escolares con representaciones más amplias y comprensivas del aprendizaje. Los siguientes relatos, dan cuenta del aporte que significa la llegada de los estudiantes migrantes a la escuela, para algunos directivos, especialmente porque se reconoce como una oportunidad para favorecer el aprendizaje de sus compañeros:

Categoría: valoración de las diferencias desde un enfoque multicultural

\begin{abstract}
Los estudiantes migrantes que hemos recibido son bien alegres y respetuosos. En realidad, ellos han sido un aporte para la escuela en todo sentido, son buenos compañeros y se esfuerzan harto por aprender, son un ejemplo para todos por su fortaleza...no es fácil llegar a un país completamente extraño y salir adelante. (Directora, caso 5)

Los estudiantes migrantes han favorecido mucho a sus compañeros, pienso que han ayudado a ampliar la mente de los demás niños. Los chiquillos se han sensibilizado respecto a lo importante que es para sus compañeros sentirse acogidos. Además, han aprendido muchas cosas nuevas, desde modismos, hasta costumbres totalmente desconocidas para ellos. (Director, caso 18)
\end{abstract}

Estos relatos, evidencian valoración hacia la presencia de estudiantes migrantes, como una oportunidad para generar aprendizaje colaborativo y avanzar en la construcción de diálogos interculturales al interior de las aulas (Echeita, 2013; Gairín, 2014; Jiménez y Fardella, 2015; Mellado y Chaucono, 2016; Sales, Fernández y Moliner, 2012). Especialmente, se valora el intercambio de saberes cotidianos entre estudiantes, como es el caso de costumbres y modismos propios del lenguaje. Estas representaciones posicionan el aprendizaje como un proceso de construcción social, propicio para el reconocimiento y valoración del otro.

\title{
4. Conclusiones
}

El desarrollo de esta investigación permite concluir que las representaciones de los líderes escolares respecto al proceso de inclusión del estudiantado migrante pueden condicionar fuertemente las oportunidades de aprendizaje de estos niños, niñas y jóvenes. En este sentido, un primer desafío para los profesionales encargados de dirigir los centros escolares, tiene relación con la necesidad de reflexionar críticamente respecto a la influencia del liderazgo como una valiosa oportunidad para propiciar la construcción de 
culturas escolares más democráticas y respetuosas de la diversidad (Fullan y RinconGallardo, 2008; Leiva, 2013).

En este sentido, es necesario superar la mirada restringida y tradicional del liderazgo escolar centrados en aspectos propios de la administración escolar, para transitar hacia liderazgos inclusivos para la justicia social (Essomba, 2006; Guzmán, 2018) centrados en de aprendizaje y bienestar de todos y todas las estudiantes. En esta línea, se requieren directivos escolares con un fuerte liderazgo ético (Giles y Cuéllar, 2017; Starratt, 2004) con capacidades para movilizar procesos de mejoramiento educativo coherentes con las necesidades e intereses del estudiantado. Como plantea, Quintriqueo y otros (2016) se precisan liderazgos para superar la cultura homogeneizadora de la escuela en la cual los estudiantes asimilar formas de pensamiento, comportamiento y aprendizaje.

En esta línea, un tercer desafío para el liderazgo, tiene relación con identificar oportunamente las necesidades formativas del profesorado en materia de inclusión, de modo de acompañar su desarrollo profesional al interior de la escuela. En coherencia con Fernández-Batanero y Hernández (2013) plantea que mejora de la calidad de la práctica docente constituye uno de los retos más importantes para avanzar hacia una educación inclusiva e intercultural, de ahí la relevancia de implementar prácticas pedagógicas que validen las diferencias culturales, como oportunidad para entablar diálogos, co-costruir saberes y valores que tributen al desarrollo de aprendizajes integrales y de calidad.

Se recomienda también, en función de favorecer el desarrollo de culturas inclusivas, contextualizar los proyectos curriculares, atendiendo a las necesidades de este nuevo escenario educativo, diverso y multicultural. La educación enfrenta el desafío de formar niños y jóvenes desde una mirada intercultural, en que éstos puedan relacionarse académica, social y afectivamente con otras personas de diferentes culturas, grupos sociales, religiones y etnias (Lehtomäki, Moate y Posti-Ahokas, 2015).

Por último, es importante reflexionar, desde el rol del líder, acerca del carácter social de la escuela, y visualizarlo como un escenario propicio para formar ciudadanos solidarios y respetuosos de lo diverso. Las escuelas no serán inclusivas por el mero hecho de asegurar el acceso y la permanencia de los niños, niñas y jóvenes, sino porque reconocen y validan las diferencias como una oportunidad para generar diálogos, saberes y valores que tributen al desarrollo de aprendizajes integrales y de calidad.

\section{Referencias}

Alavez, A. (2014). Interculturalidad: Conceptos, alcances y derecho. Ciudad de México: Editorial del GPPRD.

Anderson, S. (2010). Liderazgo directivo: Claves para una mejor escuela. Revista Psicoperspectivas, 9(2), 34-52. https://doi.org/10.5027/psicoperspectivas-Vol9-Issue2-fulltext-127

Apablaza, M. (2015). El orden en la producción de conocimiento: Normatividades en la educación chilena en torno a diversidad. Estudios Pedagógicos, 53(9), 1689-1699. https://doi.org/10.4067/S0718-07052015000300016

Arar, K. (2015). Leadership for equity and social justice in Arab and Jewish schools in Israel: Leadership trajectories and pedagogical praxis. International Journal of Multicultural Education, 17, 162-187. https://doi.org/10.18251/ijme.v17i1.938 
Barber, M. y Mourshed, M. (2007). How the world's best performing systems come out on top. Londres: McKinsey \& Company.

Bolívar, A. (2012). Justicia social y equidad escolar. Una revisión actual. Revista Internacional de Educación para la Justicia Social, 1(1), 9-45.

Bolívar, A. y Murillo, F. (2017). El efecto escuela: Un reto de liderazgo para el aprendizaje y la equidad. En J. Weinstein y G. Muñoz. (Ed.), Mejoramiento y liderazgo en la escuela: Once miradas (pp.71-112), Santiago de Chile: Ediciones Diego Portales.

Booth, T. (2010). La inclusión como marco para el desarrollo educativo: Transformar los valores en acción. En Fundación PAR (Coord.), Escuelas inclusivas un camino para construir entre todos (pp. 38-58). Buenos Aires: Fundación PAR.

Booth, T. y Ainscow, M. (2015). Guía para la evaluación y mejora de la educación inclusiva. Desarrollando el aprendizaje y la participación en los centros escolares. Madrid: OEI/FUHEM.

Bustos, R. y Gairín, J. (2017). Adaptación académica de estudiantes migrantes en contexto de frontera. Revista Calidad en Educación, 46, 193-220.

https://doi.org/10.31619/caledu.n46.7

Calvo. G. (2013). La formación de docentes para la inclusión educativa. Páginas de Educación, 6(1), $1-22$.

Calvo, M., Verdugo, M. y Amor, J. (2016). La participación familiar es un requisito imprescindible para una escuela inclusiva. Revista Latinoamericana de Educación Inclusiva, 10(1), 99-113. https://doi.org/10.4067/SO718-73782016000100006

Cerón, L., Pérez-Alvarado, M. y Poblete, R. (2017). Percepciones docentes en torno a la presencia de niños y niñas migrantes en escuelas de Santiago: Retos y desafíos para la inclusión. Revista Latinoamericana de Educación Inclusiva, 11 (2), 233-246. https://doi.org/10.4067/S0718-73782017000200015

Diez, M. (2013). Repensando la interculturalidad en educación: Aportes de la investigación socioantropológica a un campo problemático. Revista Docencia, 51, 4-17.

Dovigo, F. (2014). El tratamiento de la diversidad en las instituciones educativas. En J. Gairín (Coord.), Reflexiones y propuestas para la intervención (pp. 87-118). Madrid: Wolters Kluwer.

Echeita, G. (2013). Inclusión y exclusión educativa: De nuevo voz y quebranto. Revista Electrónica Iberoamericana sobre Calidad, Eficacia y Cambio en Educación, 11(2),100-118.

Echeita, G. y Ainscow, M. (2011). La educación inclusiva como derecho. Marco de referencia y pautas de acción para el desarrollo de una revolución pendiente. Revista de Didáctica de la Lengua y la Literatura, 12, 26-46.

Essomba, M. (2006). Liderar escuelas interculturales e inclusivas: equipos directivos y profesorado ante la diversidad cultural y la inmigración. Barcelona: Graó.

Fernández, M. P. (2018). Mapa del estudiantado extranjero en el sistema escolar chileno (2015-2017). Santiago de Chile: Centros de Estudios MINEDUC.

Fernández-Batanero, J. M. y Fernández, A. (2013). Liderazgo directivo e inclusión educativa Estudio de casos. Perfiles Educativos, 35(142), 27-41. https://doi.org/10.1016/So1852698(13)71847-6

Gairín, J. (2014). Colectivos vulnerables en la universidad. Reflexiones y propuestas para la intervención. Madrid: Wolters Kluwer Educación. 
Giles, D y Cuéllar. C. (2016). Liderazgo ético: Una forma de ser en el liderazgo. En J. Weinstein. (Ed.), Liderazgo educativo en la escuela. Nueve miradas (pp. 121-154). Santiago de Chile: Ediciones Diego Portales.

González-González, M. T. (2008). Diversidad e inclusión educativa: Algunas reflexiones sobre el liderazgo en el centro escolar. REICE. Revista Iberoamericana sobre Calidad, Eficacia y Cambio en Educación, 6(2), 82-99.

Guzmán, F. (2018). Los retos de la educación intercultural en el siglo XXI. Revista Latinoamericana de Educación Inclusiva, 12(1), 199-212. https://doi.org/10.4067/S071873782018000100199

Hargreaves, A. y Fink, D. (2008). El liderazgo sostenible: Siete principios para el liderazgo en centros educativos innovadores. Madrid: Morata.

Hargreaves, A. y Fullan, M. (2012). Profesional capital. Transforming teaching in every school. Nueva York, NY: Teachers College Press.

Hernández-Castilla, R., Euán, R. e Hidalgo, N. (2013). Prácticas del liderazgo escolar implicado en la promoción de la justicia social: Estudio de un caso en educación secundaria. Profesorado. Revista de Currículum y Formación del Profesorado, 17(2), 263-280.

Instituto Nacional de Derechos Humanos. (2018). Diagnóstico sobre la migración en la región de La Araucanía. Avances y brechas en vivienda y situación laboral. Temuco: Instituto de Estudios Indígenas e Interculturales.

Jiménez, F. (2014). Modelos de gestión de la diversidad cultural para la escolarización de alumnado inmigrante en las escuelas chilenas: Desafíos para la interculturalidad actual. Estudios Pedagógicos, 40(2), 409-426. https://doi.org/10.4067/S0718-07052014000300024

Jiménez, F. y Fardella, C. (2015). Diversidad y rol de la escuela: Discursos del profesorado en contextos educativos multiculturales en clave migratoria. Revista Mexicana de Investigación Educativa, 20(65), 419-441.

Jiménez, F., Aguilera, M., Valdés, R. y Hernández, M. (2017). Migración y escuela: Análisis documental en torno a la incorporación de inmigrantes al sistema educativo chileno. Revista Psicoperspectivas, 16(1), 105-116.

Joiko, S. y Vásquez, A. (2016). Acceso y elección escolar de familias migrantes en Chile: No tuve problemas porque la escuela es abierta, porque acepta muchas nacionalidades. Calidad en la Educación, 45, 132-173. https://doi.org/10.4067/S0718-45652016000200005

Lehtomäki, E., Moate, J. y Posti-Ahokas, H. (2015). Global connectedness in higher education: Student voices on the value of cross-cultural learning dialogue. Studies in Higher Education, 5079, 1-17.

Leithwood, K. y Riehl, C. (2005). What we know about successful leadership. EnW. Firetone y C. Riehl (Eds.), A new agenda: Directions for researshon educational leadership (pp. 22-47). Nueva York, NY: Teachers College Press.

Leithwood, K., Day, C., Sammons, P., Harris, A. y Hopkins, D. (2006). Successful school leadership. What it is and how it influences pupil learning. Londres: National College for School Leadership.

Leiva, J. (2013). De la integración a la inclusión: Evolución y cambio en la mentalidad del alumnado universitario de educación especial en un contexto universitario español. Revista Actualidades Investigativas en Educación, 13(3), 1-27. 
Mampaey, J. y Zanoni, P. (2015). Reproducing monocultural education: Ethnic majority staff's discursive constructions of monocultural school practices. British Journal of Sociology of Education, 3, 1-19.

Mansilla, J. y Beltrán, J. (2016). Racionalidad instrumental y prácticas de gestión en jefes técnicos de liceos de La Araucanía. Revista de Investigación Educativa, 34(1), 151-165. https://doi.org/10.6018/rie.34.1.218041

Martínez, L., Diez, M., Novaro, G. y Groisman, L. (2015). Migración e interculturalidad: Perspectivas de derechos y política educativa. Boletín de Antropología y Educación, 6(9), 5762.

Mellado, M. E. y Chaucono J. C. (2016). Liderazgo pedagógico para reestructurar creencias docentes y mejorar prácticas de aula en contexto mapuche. Revista Electrónica Educare, 20(1), 1-18. https://doi.org/10.15517/revedu.v41i1.21597

Mellado, M. E., Chaucono, J. C., Hueche, M. y Aravena, O. (2017). Percepciones sobre la educación inclusiva del profesorado de una escuela con programa de integración escolar. Revista Educación, 41(1), 1-14.

MINEDUC. (2015). Marco para la buena dirección y el liderazgo escolar. Santiago de Chile: Centro de perfeccionamiento, experimentación e investigaciones pedagógicas.

MINEDUC. (2016a). Orientaciones para la construcción de comunidades educativas inclusivas. Santiago de Chile: División de Educación General.

MINEDUC. (2016b). Niños y niñas migrantes: Trayectorias de inclusión educativa en escuelas de la Región Metropolitana. Santiago de Chile: Superintendencia de Educación.

MINEDUC. (2017a). El primer gran debate de la reforma educacional: Ley de inclusión escolar. Santiago de Chile: Programa de las Naciones Unidas para el Desarrollo.

MINEDUC. (2017b). Orientaciones técnicas para la Inclusión educativa de estudiantes extranjeros, Santiago de Chile: División de Educación General.

Mintrop, R. y Órdenes, M. (2017). Motivación laboral de los docentes: Un desafío directivo de alto impacto. En J. Weinstein y G. Muñoz. (Ed.), Mejoramiento y liderazgo en la escuela: Once miradas (pp. 295-332). Santiago de Chile: Ediciones Diego Portales.

Muntaner, J. (2010) De la integración a la inclusión: un nuevo modelo educativo. En P. Arnaiz, M. Hurtado y F. Soto (Coords.), 25 Años de integración escolar en España: Tecnología e inclusión en el ámbito educativo, laboral y comunitario. Murcia: Consejería de Educación, Formación y Empleo.

Murillo, F. J. y Duk, C. (2016). Segregación escolar e inclusión. Revista Latinoamericana de Educación Inclusiva, 1O(2), 11-13. https://doi.org/10.4067/So718-73782016000200001

Murillo, F. J. y Duk, C. (2017). El ODS 4 (y el 16) como meta para los próximos años. Revista Latinoamericana de Educación Inclusiva, 11(2), 11-13. https://doi.org/10.4067/S0718-73782017000200001

Murillo, F. J. y Hernández-Castilla, R. (2011). Hacia un concepto de justicia social. REICE. Revista Iberoamericana sobre Calidad, Eficacia y Cambio en Educación, 9(4), 7-23.

Murillo, F. J., Krichesky, G., Castro, A. y Hernández-Castilla, R. (2010). Liderazgo para la inclusión escolar y la justicia social. Aportaciones de la investigación. Revista Latinoamericana de Patología, 4(1),169-187.

Ossa, C., Castro, F., Castañeda, M. y Castro, J. (2014). Cultura y liderazgo escolar: Factores claves para el desarrollo de la inclusión educativa. Revista Actualidades Investigativas en Educación, $14(3), 1-23$. 
Quintriqueo, S., Morales, S., Quilaqueo, D. y Arias, K. (2016). Interculturalidad para la formación inicial docente: Desafio para construir un diálogo intercultural. Temuco: Universidad Católica de Temuco.

Riedemann, A. y Stefoni, C. (2015). Sobre el racismo, su negación, y las consecuencias para una educación anti-racista en la enseñanza secundaria chilena. Polis Revista Latinoamericana, 42, 191-216.

Rincón-Gallardo, S. y Fullan, M. (2016). Essential features of effective networks in education. Journal of Professional Capital and Community, 1(1), 5-23. https://doi.org/10.1108/JPCC-092015-0007

Rojas, M. T. y Armijo, M. (2016). Qué es la inclusión escolar: Distintas perspectivas en debate. Cuadernos de Educación, 75, 1-11.

Rodríguez-Ruiz, B., Martínez-González, R. y Rodrigo-López. M. (2016). Dificultades de las familias para participar en los centros escolares. Revista Latinoamericana de Educación Inclusiva, 1O(1), 79-98. https://doi.org/10.4067/So718-73782016000100005

Ryan, J. (2016). Un liderazgo inclusivo para las escuelas. En J. Weinstein. (Ed.), Liderazgo educativo en la escuela. Nueve miradas (pp. 177-204). Santiago de Chile: Ediciones Diego Portales.

Sales, M., Fernández, R. y Moliner, O. (2012). Escuela intercultural inclusiva: Estudio de caso sobre los procesos de autoevaluación. Revista de Educación, 358, 153-173.

Sánchez, H. y Gil, I. (2015). Análisis interseccional y enfoque intercultural en el estudio de la ciudadanía y la participación. Consideraciones epistemológicas. Diálogo Andino, 47, 143-149. https://doi.org/10.4067/S0719-26812015000200015

Starratt, R. (2004). Ethical leadership, San Francisco, CA: Jossey-Bass.

Sun, J. y Leithwood, K. (2015). Leadership effect on student learning mediated by teacher emotions. Societties, 5(3), 566-582. https://doi.org/10.3390/soc5030566

Tintore-Espuny, M. (2018). Líderes educativos y justicia social. Un estudio comparativo. Revista Perspectiva Educacional, 57(2), 100-122.

Tubino, F. (2005). La praxis de la interculturalidad en los estados nacionales latinoamericanos. Cuadernos Interculturales, 3(5), 83-96.

Tubino, F. (2011). El nivel epistémico de los conflictos interculturales. Revista Electrónica Construyendo Nuestra Interculturalidad, 7(6), 1-14.

\section{Breve CV de los autores}

\section{Omar Aravena Kenigs}

Profesor de Educación Física de la Universidad de Los Lagos, Osorno; Magíster en Educación Física de la Universidad de la Frontera, Temuco; Magíster en Gestión Escolar de la Universidad Católica de Temuco; Candidato Doctoral en Formación del Profesorado y Análisis de la Práctica Educativa de la Universidad de Extremadura, España. Se desempeña como profesional de la Facultad de Educación de la Universidad Católica de Temuco e investigador del Centro de Desarrollo de Liderazgo Educativo (CEDLE). ORCID ID: https://orcid.org/0000-0002-0896-6657. Email: oaravena@uct.cl 


\section{Paula Riquelme Bravo}

Profesora de Educación Diferencial Mención Retardo Mental; Magíster en Educación Mención Evaluación; Doctora en Educación de la Universidad de Barcelona; Máster en Psicomotricidad. (ICSE. España); Especialista en Psicodiagnóstico y Estimulación Temprana, Universidad de Comillas, España. Actualmente es Académica y Directora General de Docencia de la Facultad de Educación y Humanidades de la Universidad Andrés Bello, Santiago. ORCID ID: https://orcid.org/0000-0001-5914-4015. Email: paula.riquelme@unab.cl

\section{Ma Elena Mellado Hernández}

Profesora de Educación General Básica de la Pontificia Universidad Católica de Chile; Magíster en Informática Educativa de la Universidad de La Frontera, Temuco; Doctora en Formación del Profesorado de la Universidad de Extremadura España. Actualmente se desempeña como académica y es Directora del Programa de Magíster en Gestión Escolar de la Universidad Católica de Temuco y Directora del Proyecto del Centro de Desarrollo de Liderazgo Educativo "Transformación de Redes de Mejoramiento Escolar en Comunidades de Aprendizaje”. ORCID ID: https://orcid.org/0000-0003-2039-5145. Email:mmellado@uct.cl

\section{Carolina Villagra Bravo}

Profesora de Educación General Básica de la Universidad Católica de Temuco; Magíster en Educación con Mención en Evaluación Educativa de la Universidad de La Frontera; Candidata Doctoral en Formación del Profesorado y Análisis de la Práctica Educativa de la Universidad de Extremadura España. Actualmente se desempeña como Académica de la Universidad Católica de Temuco e investigadora del Centro de Desarrollo de Liderazgo Educativo (CEDLE). Es coordinadora del Proyecto CEDLE "Transformación de Redes de Mejoramiento Escolar en Comunidades de Aprendizaje”. ORCID ID: https://orcid.org/0000-0002-5428-2555. Email: cvillagra@uct.cl 